\title{
4D Lattice Flower Constellations
}

\author{
David Arnas $^{\mathrm{a}, *}$, Daniel Casanova ${ }^{\mathrm{b}}$, Eva Tresaco ${ }^{\mathrm{b}}$ \\ ${ }^{a}$ Massachusetts Institute of Technology, Cambridge, MA 02139, USA \\ ${ }^{\mathrm{b}}$ Centro Universitario de la Defensa - Zaragoza, Crta. Huesca s/n, 50090 Zaragoza, Spain
}

Received 23 January 2020; received in revised form 7 April 2020; accepted 11 April 2020

\begin{abstract}
4D Lattice Flower Constellations is a new constellation design framework, based on the previous 2D and 3D Lattice theories of Flower Constellations, that focus on the generation of constellations whose satellites can have different semi-major axis and still present a constellation structure that is maintained during the dynamic of the system. This situation can arise when dealing with satellites with very different instruments, or when it is of interest to coordinate two different constellations. In that sense, 4D Lattice Flower Constellations constitutes the most general representation of the Flower Constellation formulation. In addition, the effects of the $J_{2}$ perturbation are taken into account in order to generate distributions that maintain their initial design configuration under this perturbation for longer periods of time with a low fuel budget. Finally, examples of application are presented, showing the possibilities in satellite constellation design of this new approach.
\end{abstract}

(C) 2020 COSPAR. Published by Elsevier Ltd. All rights reserved.

Keywords: Space mechanics; Satellite constellation design; Number theory; Optimization techniques; Mega-constellations

\section{Introduction}

Satellites orbiting the Earth provide a great number of possibilities and include a variety of missions such as Earth and space observation, telecommunications or global positioning systems. These possibilities can be increased by the use of groups of satellites that work cooperatively to achieve a common mission, that is, a satellite constellation. The advantages of using satellite constellations are numerous, including the gain in performance of the system as a whole, or the reduction of the costs associated with the mission. However, the study of several satellites at the same time, and more importantly, the relations that appear in the internal structure of the constellation, increases not only the complexity of the problem to solve, but also the

\footnotetext{
* Corresponding author.

E-mail addresses: arnas@mit.edu (D. Arnas), casanov@unizar.es (D. Casanova), etresaco@unizar.es (E. Tresaco).
}

available possibilities in the design of constellation configurations.

Satellite constellation design has been since its beginning a complex process due to the lack of established models for generating and studying satellite constellations under any situation, leading to the need of performing specific studies for each particular mission. Fortunately, in the last decades, several satellite constellation design methodologies have appeared to minimize this issue. Some very well known examples of that are Walker Constellations (Walker, 1984) for circular orbits or the design of Draim (Draim, 1987) for elliptic orbits, but there are many others (Ulybyshev, 2008; Lo, 1999; Arnas et al., 2017b).

In 2004, the Flower Constellation Theory (Mortari et al., 2004; Mortari and Wilkins, 2008; Wilkins and Mortari, 2008) was presented, including in its formulation circular and elliptic orbits and containing the former designs of Walker and Draim as particular cases of this design methodology. The theory was later improved by the 2D Lattice (Avendaño et al., 2013) and 3D Lattice 
(Davis et al., 2013) theories which simplified the formulation and made the configuration independent of any reference frame. One of the most important properties of Lattice Flower Constellations is that the structure generated in the constellation presents a high number of symmetries, meaning that the configuration that each satellite observes is equivalent to the others of the constellation. This property makes this methodology of design interesting for many applications, especially global coverage, telecommunications and global positioning systems (Casanova et al., 2014a). Later, Lattice Flower Constellations were generalized with Necklace Flower Constellations(Arnas, 2018; Casanova et al., 2014b; Arnas et al., 2017a; Arnas et al., 2017c; Arnas et al., 2017d), which allowed to expand the number of possibilities of design by defining a fictitious constellation from where a subset of satellites were selected in such a way that the distribution properties of uniformity and symmetry were preserved.

In this work, we introduce the 4D Lattice Flower Constellations (4D-LFC) as a general methodology for designing satellite constellations. The most important particularity of $4 \mathrm{D}-\mathrm{LFC}$ is that satellites of the constellation are not required to have the same semi-major axis. Examples of these kind of constellations and their possible applications can be seen in the designs of Rider (1985) and Palmerini (1998). This situation allows to generate constellations whose satellites have very different instruments, or to coordinate completely different constellations. In fact, 4D-LFC can be also regarded as a coordination of different 2D and 3D Lattice Flower Constellations that have different semi-major axis but for some reason, they are required to work cooperatively.

The idea behind 4D-LFC is to define a periodic constellation dynamic in such a way that its resultant configuration presents the highest number of symmetries, both in time and geometrically, in a similar way as the $2 \mathrm{D}$ and 3D Lattice Flower Constellations were defined. That way, and based on the Lattice Theory, the 4D-LFC are able to provide all the uniformly distributed constellations that can be arranged with a given set of satellites (Arnas, 2018).

In this work we proceed as follows. First, a summary of the previous 2D and 3D Lattice Flower Constellations methodologies is presented. Second, the 4D Lattice Flower Constellation theory is introduced, showing also its relation with the former methodologies. Third, the effects of the $J_{2}$ perturbation are included in the design in order to maintain the structure of the constellation over longer periods of time with a low fuel budget. Fourth, a simple example of application is presented where we show a possible use of this methodology for telecommunications and deep space observation. Finally, an example of application of this methodology to the design of mega-constellations is presented.

\section{Preliminaries}

\subsection{D Lattice Flower Constellations}

A 2D Lattice Flower Constellation (Avendaño et al., 2013) (2D-LFC) is described by nine parameters: three integers and six continuous parameters. The first three parameters are the number of inertial orbits $\left(N_{o}\right)$, the number of satellites per orbit $\left(N_{s o}\right)$ and the configuration number $\left(N_{c}\right)$, which is a parameter that satisfies $N_{c} \in\left\{0, \ldots, N_{o}-1\right\}$ and governs the phasing of the constellation. In particular, the location of the satellites in a 2D-LFC corresponds to a lattice in the $(\Omega, M)$-space (Avendaño and Mortari, 2009), that is, a space generated in the orbital variables right ascension of the ascending node $\Omega$ and mean anomaly $M$ of all the satellites of the constellation in a given instant. The $(\Omega, M)$-space can be also regarded as a $2 \mathrm{D}$ torus (both axes, $\Omega$ and $M$, are modulo $2 \pi$ ) where the points represented coincide with the solutions of the following system of equations:

$$
\left(\begin{array}{cc}
N_{o} & 0 \\
N_{c} & N_{s o}
\end{array}\right)\left(\begin{array}{c}
\Delta \Omega_{i j} \\
\Delta M_{i j}
\end{array}\right)=2 \pi\left(\begin{array}{c}
i-1 \\
j-1
\end{array}\right)
$$

where $i=\left\{1, \cdots, N_{o}\right\}, j=\left\{1, \cdots, N_{s o}\right\}$, and $\Delta \Omega_{i j}$ and $\Delta M_{i j}$ represent the satellite distribution in the right ascension of the ascending node and the mean anomaly with respect to a reference satellite. Indexes $(i, j)$ represent the $j$-th satellite on the $i$-th orbital plane. Note that this system of equations is derived from the Hermite Normal Form of the lattice, which is the minimum representation of a lattice in a $2 \mathrm{D}$ distribution (Avendaño et al., 2013).

On the other hand, the other six parameters are the semi-major axis $(a)$, the eccentricity $(e)$, the inclination (inc) and the argument of perigee $(\omega)$ (which are the same for all the satellites of the constellation), and the longitude of the ascending node and the initial mean anomaly of the first satellite of the constellation, that is, $\Omega_{11}$ and $M_{11}$ (and which define a reference for the constellation).

One example of 2D-LFC is Galileo (Casanova et al., 2014a). This constellation can be represented by the 2DLFC formulation using as distribution parameters $N_{o}=3, N_{s o}=7$, and $N_{c}=2$. Fig. 1 shows the $(\Omega, M)$ space representation (Avendaño et al., 2013) of the distribution, while Fig. 2 presents the inertial distribution of the constellation.

\subsection{D Lattice Flower Constellations}

3D Lattice Flower Constellations (Davis et al., 2013) (3D-LFC) is a satellite constellation design methodology in which satellites are distributed in several inertial orbits, where each satellite has in general a different value of its mean anomaly, argument of perigee and right ascension 


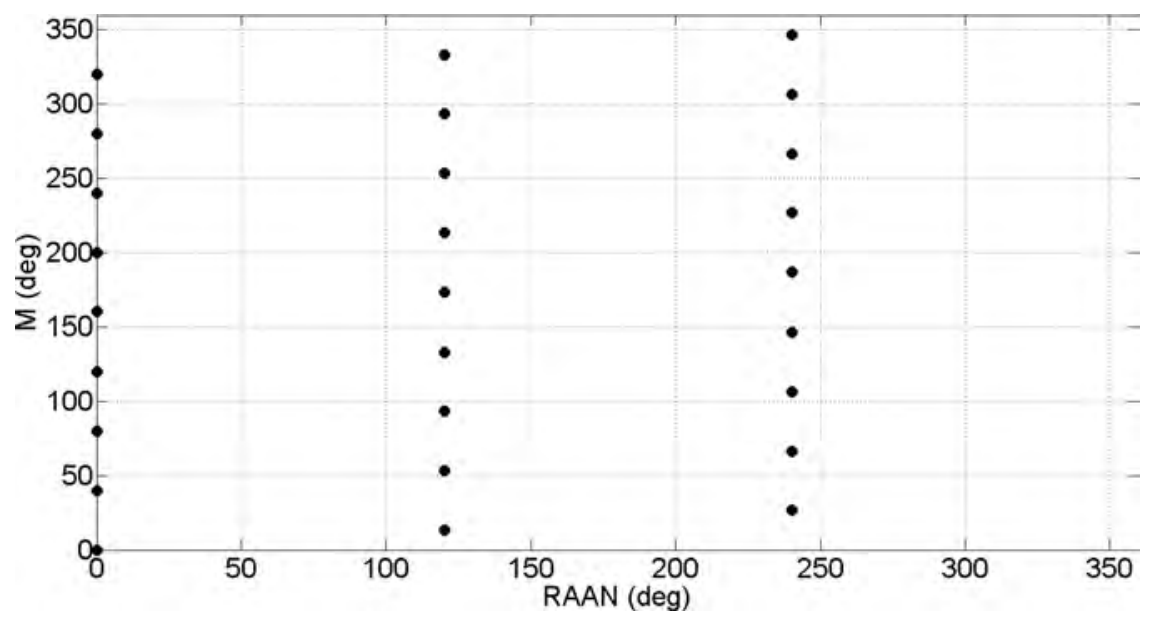

Fig. 1. $(\Omega, M)$-space representation of Galileo constellation.

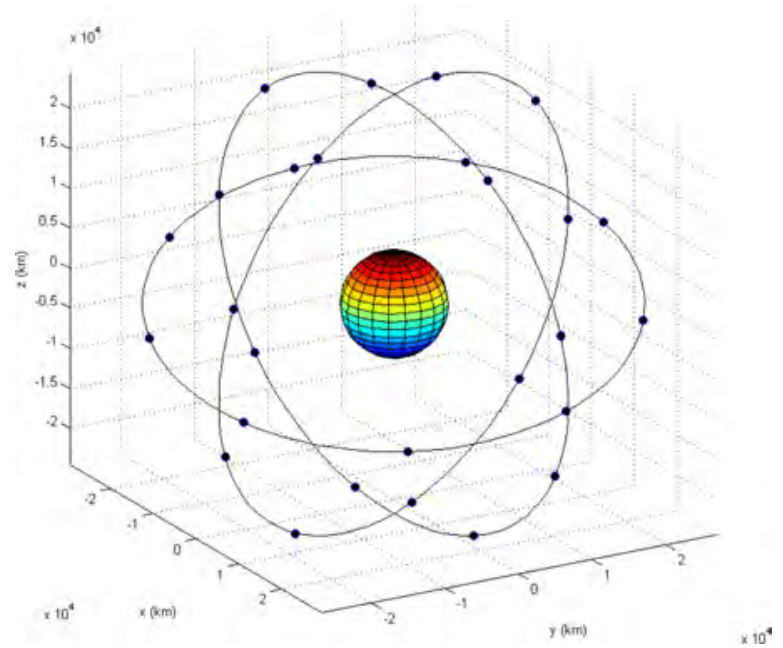

Fig. 2. Inertial representation of Galileo constellation.

of the ascending node. In addition, the satellites of the constellation have the same semi-major axis, eccentricity and inclination. This technique was originally devised for the design of elliptical constellations at any inclination under the $J_{2}$ perturbation. That way, an additional degree of freedom to orbit design is provided compared to the $2 \mathrm{D}$ formulation, the argument of perigee and the ability to design constellations at any inclination. Moreover, as in the case of 2D Lattice Flower constellations, the distributions present symmetric and uniform configurations in their lattices that are maintained over time.

Following Davis et al. (2013), a 3D Lattice Flower Constellation can be described by the use of the Hermite Normal Form. In this case, the Hermite Normal Form is composed by six integers, three in the diagonal of the matrix and the other three in the inferior part of the matrix. The integers in the diagonal are the number of orbital planes of the constellation $\left(N_{o}\right)$, the number of different arguments of perigee in each orbital plane $\left(N_{\omega}\right)$, and the number of satellites in each orbit $\left(N_{s o}\right)$. The other three parameters are configuration numbers $\left(N_{c_{1}}, N_{c_{2}}, N_{c_{3}}\right)$ defined as follows: $\quad N_{c_{1}} \in\left\{0, \ldots, N_{o}-1\right\}, N_{c_{2}} \in$ $\left\{0, \ldots, N_{\omega}-1\right\}$ and $N_{c_{3}} \in\left\{0, \ldots, N_{o}-1\right\}$.

The expression that summarizes the distribution of the satellites in a 3D Lattice Flower Constellation is:

$$
\left(\begin{array}{ccc}
N_{o} & 0 & 0 \\
N_{c_{3}} & N_{\omega} & 0 \\
N_{c_{1}} & N_{c_{2}} & N_{s o}
\end{array}\right)\left(\begin{array}{c}
\Delta \Omega_{i j k} \\
\Delta \omega_{i j k} \\
\Delta M_{i j k}
\end{array}\right)=2 \pi\left(\begin{array}{c}
i-1 \\
k-1 \\
j-1
\end{array}\right) ;
$$

where $\Delta \Omega_{i j k}$ is the distribution in the right ascension of the ascending node of the constellation, $\Delta \omega_{i j k}$ is the distribution of the argument of perigee, and $\Delta M_{i j k}$ is the distribution of the mean anomaly, with respect to a reference satellite of the constellation with parameters $\Omega_{111}, \omega_{111}$ and $\quad M_{111}$. Moreover, the sub-indexes $i=\left\{1, \ldots, N_{o}\right\}, j=\left\{1, \ldots, N_{s o}\right\}$, and $k=\left\{1, \ldots, N_{w}\right\}$, represent the position of a satellite in the orbital plane $i$, with the argument of perigee $k$ and the mean anomaly $j$. Note also that the values of $\Delta \Omega_{i j k}, \Delta \omega_{i j k}$ and $\Delta M_{i j k}$ represent three angles and thus are defined in the range $[0,2 \pi]$ (showing also a modular behavior).

\section{4D Lattice Flower Constellations}

\subsection{Motivation}

2D-LFC and 3D-LFC focus on the generation of constellations whose satellites have very similar mission requirements and instruments, and thus, it is possible to locate satellites in orbits with the same semi-major axis. However, there are cases in which it is of interest that satellites with very different properties are able to cooperate in their missions, a situation that is increasing each passing year in the space industry.

For instance, imagine that we want to perform deep space observation at a high altitude but, due to satellite design or orbit geometry, it is not feasible for these satellites to perform a direct communication with their ground stations. In this case, it is possible to solve this problem by 
locating an auxiliary constellation at a lower altitude whose mission is to perform the communication between the former satellites and their ground stations. Under these circumstances, a proper constellation design should provide a uniform revisiting time between satellites and a periodic behavior to ease the control strategy and telecommunications of this system. These are the properties sought by 4D-LFC.

Another situation where coordination between satellites in different semi-major axes is important is when dealing with the problem of signal interference between satellite antennas. In that regard, multiple missions have experienced this problem in the past and have solved them by a coordinated control strategy. However, and due to the increasing number of satellites launched, this problem is expected to worsen in the following years. Therefore, it is important to define a constellation design formulation specially devised to study this coordinated dynamic. In that sense, 4D-LFC represents a first step in that regard that focus on the uniform and periodic satellite distributions.

In essence, 4D-LFC deals with the problem of designing satellite constellations in different semi-major axes in such a way that the properties of uniformity, symmetry and periodicity are maintained in the distribution. Particularly, 4DLFC can be regarded as the combination of a set of different 2D-LFC and 3D-LFC that aim to work under a common coordinated dynamic.

\subsection{Formulation}

4D-LFC are based on the idea of performing a uniform distribution of satellites in four variables: the semi-major axis $(a)$, the right ascension of the ascending node $(\Omega)$, the argument of perigee $(\omega)$ and the mean anomaly $(M)$. In order to obtain all possible uniform distributions (Arnas, 2018), it is required to define a lattice distribution in a 4D space in a similar manner as it was done in the 2D-LFC (Avendaño et al., 2013) and 3D-LFC (Davis et al., 2013). However, and due to the nature of the new distribution variable (the semi-major axis) this lattice must be defined in a slightly different manner. The reasons for that lay in two properties. First, the semi-major axis $(a)$ does not have a modular nature unlike the angles mean anomaly $(M)$, argument of perigee $(\omega)$ and right ascension of the ascending node $(\Omega)$. Second, we are seeking constellation structures that are maintained over time, and thus, we are interested in constellations whose dynamic presents periodicity.

Therefore, and in order to solve this difficulty, an alternative process is followed. First, a $4 \mathrm{D}$ space is defined where each variable $(V)$ in this space ranges in $[0,1]$. This allows to define a completely uniform distribution using the Lattice Theory. Second, a transformation of this configuration is performed, leading to an equivalent distribution based completely on integer positions. In this equivalent distribution each position in a given dimension represents one of the possible combinations that the distribution variables $a, \Omega, \omega$ and $M$ can acquire for each satellite of the constellation. Finally, these positions are related with the final distribution variables, obtaining the real constellation configuration. In the following lines this process is explained in more detail.

The first step in the definition process is to generate a set of four distribution variables, each one in a different dimension, in the range $[0,1]:\left\{V^{a}, V^{\Omega}, V^{\omega}, V^{M}\right\}$, where $V^{a}$ is related to the distribution in the semi-major axis, $V^{\Omega}$ is related to the right ascension of the ascending node, $V^{\omega}$ is related to the argument of perigee, and $V^{M}$ is related to the initial mean anomaly of a satellite of the constellation. It is important to remember that $\left\{V^{a}, V^{\Omega}, V^{\omega}, V^{M}\right\}$ do not represent the real values of the semi-major axis, the right ascension of the ascending node, the argument of perigee or the initial mean anomaly. Instead, these variables define a set of possible values for each orbital parameter that are used to generate the real distribution of the constellation. That way, a 4D lattice can be easily defined using the Hermite Normal Form:

$$
\left(\begin{array}{cccc}
L_{a} & 0 & 0 & 0 \\
L_{\Omega a} & L_{\Omega} & 0 & 0 \\
L_{\omega a} & L_{\omega \Omega} & L_{\omega} & 0 \\
L_{M a} & L_{M \Omega} & L_{M \omega} & L_{M}
\end{array}\right)\left(\begin{array}{c}
V_{i j k r}^{a} \\
V_{i j k r}^{\Omega} \\
V_{i j k r}^{\omega} \\
V_{i j k r}^{M}
\end{array}\right)=\left(\begin{array}{c}
r \\
i \\
k \\
j
\end{array}\right) ;
$$

where $L_{a}$ is the number of different semi-major axes, $L_{\Omega}$ is the number of orbital planes with a given semi-major axis, $L_{\omega}$ is the number of orbits per plane with a given semimajor axis, and $L_{M}$ is the number of satellites per orbit. These parameters determine the size of the constellation. In particular, the total number of satellites that are generated is $N_{s a t}=L_{a} L_{\Omega} L_{\omega} L_{M}$. On the other hand, the configuration numbers $\left(L_{\Omega a}, L_{\omega a}, L_{M a}, L_{\omega \Omega}, L_{M \Omega}\right.$, and $\left.L_{M \omega}\right)$ determine how the relative distribution is shifted within the different semi-major axes (with $L_{\Omega a}, L_{\omega a}$, and $L_{M a}$ ), different orbital planes (with $L_{M \Omega}$ and $L_{\omega \Omega}$ ), and different orbits (with $\left.L_{M \omega}\right)$. Moreover, and in order to avoid duplicities in the constellation definition, some constraints must be imposed (Arnas, 2018). First, the parameters of distribution must be defined as $r=\left\{1, \ldots, L_{a}\right\}, i=\left\{1, \ldots, L_{\Omega}\right\}, k=\left\{1, \ldots, L_{\omega}\right\}$ and $j=\left\{1, \ldots, L_{M}\right\}$ which name each satellite of the constellation. Second, the configuration numbers must present these constraints: $L_{\Omega a} \in\left\{0, \ldots, L_{a}-1\right\}, L_{\omega a} \in\{0, \ldots$, $\left.L_{a}-1\right\}, L_{\omega \Omega} \in\left\{0, \ldots, L_{\Omega}-1\right\}, L_{M a} \in\left\{0, \ldots, L_{a}-1\right\}, L_{M \Omega} \in$ $\left\{0, \ldots, L_{\Omega}-1\right\}$ and $L_{M \omega} \in\left\{0, \ldots, L_{\omega}-1\right\}$.

Note that as opposed to what happens in 2D-LFC and 3D-LFC, Eq. (3) cannot be used directly to generate the variables of the satellites of the constellation. Instead, we have to proceed in a slightly different manner. By expanding Eq. (3), the values of the distribution variables can be obtained: 


$$
\begin{aligned}
V_{i j k r}^{a}= & \frac{1}{L_{a}} r \\
V_{i j k r}^{\Omega}= & \frac{1}{L_{\Omega}} i-\frac{L_{\Omega a}}{L_{a} L_{\Omega}} r \\
V_{i j k r}^{\omega}= & \frac{1}{L_{\omega}} k-\frac{L_{\omega \Omega}}{L_{\omega} L_{\Omega}} i-\frac{1}{L_{\omega}}\left(\frac{L_{\omega a}}{L_{a}}-\frac{L_{\omega \Omega}}{L_{\Omega}} \frac{L_{\Omega a}}{L_{a}}\right) r \\
V_{i j k r}^{M}= & \frac{1}{L_{M}} j-\frac{L_{M \omega}}{L_{M} L_{\omega}} k-\frac{1}{L_{M}}\left(\frac{L_{M \Omega}}{L_{\Omega}}-\frac{L_{M \omega}}{L_{\omega}} \frac{L_{\omega \Omega}}{L_{\Omega}}\right) i- \\
& \frac{1}{L_{M}}\left(\frac{L_{M a}}{L_{a}}-\frac{L_{M \Omega}}{L_{\Omega}} \frac{L_{\Omega a}}{L_{a}}-\frac{L_{M \omega}}{L_{\omega}}\left(\frac{L_{\omega a}}{L_{a}}-\frac{L_{\Omega a}}{L_{a}} \frac{L_{\omega \Omega}}{L_{\Omega}}\right)\right) r,
\end{aligned}
$$

and since, as said before, the distribution variables are subjected to modular arithmetic (modulo 1), Eq. (4) can be rewritten as:

$$
\begin{aligned}
V_{i j k r}^{a}= & \bmod \left[\frac{1}{L_{a}} r, 1\right] \\
V_{i j k r}^{\Omega}= & \bmod \left[\frac{1}{L_{\Omega}}\left(i-\frac{L_{\Omega a}}{L_{a}} r\right), 1\right] \\
V_{i j k r}^{\omega}= & \bmod \left[\frac{1}{L_{\omega}}\left(k-\frac{L_{\omega \Omega}}{L_{\Omega}} i-\left(\frac{L_{\omega a}}{L_{a}}-\frac{L_{\omega \Omega}}{L_{\Omega}} \frac{L_{\Omega a}}{L_{a}}\right) r\right), 1\right] \\
V_{i j k r}^{M}= & \bmod \left[\frac { 1 } { L _ { M } } \left(j-\frac{L_{M \omega}}{L_{\omega}} k-\left(\frac{L_{M \Omega}}{L_{\Omega}}-\frac{L_{M \omega}}{L_{\omega}} \frac{L_{\omega \Omega}}{L_{\Omega}}\right) i-\right.\right. \\
& \left.\left.\left(\frac{L_{M a}}{L_{a}}-\frac{L_{M \Omega}}{L_{\Omega}} \frac{L_{\Omega a}}{L_{a}}-\frac{L_{M \omega}}{L_{\omega}}\left(\frac{L_{\omega a}}{L_{a}}-\frac{L_{\Omega a}}{L_{a}} \frac{L_{\omega \Omega}}{L_{\Omega}}\right)\right) r\right), 1\right]
\end{aligned}
$$

where, in order to simplify notation, we denote $\bmod [a, b]$ to the value of $a$ modulo $b$. Now, we aim to obtain a set of distribution variables based on integer numbers, which will correspond with the different values of $a, \Omega, \omega$ and $M$ of the satellites of the constellation. In order to do that, we multiply the first expression from Eq. (5) by $L_{a}$, the second by $L_{a} L_{\Omega}$, the third by $L_{a} L_{\Omega} L_{\omega}$, and the fourth by $L_{a} L_{\Omega} L_{\omega} L_{M}$, leading to the following set of equations:

$$
\begin{aligned}
L_{a} V_{i j k r}^{a}= & \bmod \left[r, L_{a}\right], \\
L_{\Omega} L_{a} V_{i j k r}^{\Omega}= & \bmod \left[L_{a} i-L_{\Omega a} r, L_{\Omega} L_{a}\right], \\
L_{\omega} L_{\Omega} L_{a} V_{i j k r}^{\omega}= & \bmod \left[L_{\Omega} L_{a} k-L_{\omega \Omega} L_{a} i-\right. \\
& \left.\left(L_{\omega a} L_{\Omega}-L_{\omega \Omega} L_{\Omega a}\right) r, L_{\omega} L_{\Omega} L_{a}\right], \\
L_{M} L_{\omega} L_{\Omega} L_{a} V_{i j k r}^{M}= & \bmod \left[L_{\omega} L_{\Omega} L_{a} j-L_{M \omega} L_{\Omega} L_{a} k-\right. \\
& \left(L_{M \Omega} L_{\omega} L_{a}-L_{M \omega} L_{\omega \Omega} L_{a}\right) i- \\
& \left(L_{M a} L_{\omega} L_{\Omega}-L_{M \Omega} L_{\Omega a} L_{\omega}-\right. \\
& \left.\left.L_{M \omega}\left(L_{\omega a} L_{\Omega}-L_{\Omega a} L_{\omega \Omega}\right)\right) r, L_{M} L_{\omega} L_{\Omega} L_{a}\right] .
\end{aligned}
$$

Then, we define a new set of variables $\left\{\mathscr{N}^{a}, \mathscr{N}^{\Omega}, \mathscr{N}^{\omega}, \mathscr{N}^{M}\right\}$ which relate to the original one through these expressions:

$$
\begin{aligned}
& \mathscr{N}_{i j k r}^{a}=L_{a} V_{i j k r}^{a}, \\
& \mathscr{N}_{i j k r}^{\Omega}=L_{\Omega} L_{a} V_{i j k r}^{\Omega}, \\
& \mathscr{N}_{i j k r}^{\omega}=L_{\omega} L_{\Omega} L_{a} V_{i j k r}^{\omega}, \\
& \mathscr{N}_{i j k r}^{M}=L_{M} L_{\omega} L_{\Omega} L_{a} V_{i j k r}^{M},
\end{aligned}
$$

which introduced in Eq. (6), they allow us to obtain:

$$
\begin{aligned}
\mathscr{N}_{i j k r}^{a}= & \bmod \left[r, L_{a}\right], \\
\mathscr{N}_{i j k r}^{\Omega}= & \bmod \left[L_{a} i-L_{\Omega a} r, L_{\Omega} L_{a}\right], \\
\mathscr{N}_{i j k r}^{\omega}= & \bmod \left[L_{\Omega} L_{a} k-L_{\omega \Omega} L_{a} i-\left(L_{\omega a} L_{\Omega}-L_{\omega \Omega} L_{\Omega a}\right) r, L_{\omega} L_{\Omega} L_{a}\right], \\
\mathscr{N}_{i j k r}^{M}= & \bmod \left[L_{\omega} L_{\Omega} L_{a} j-L_{M \omega} L_{\Omega} L_{a} k-\left(L_{M \Omega} L_{\omega} L_{a}-L_{M \omega} L_{\omega \Omega} L_{a}\right) i-\right. \\
& \left(L_{M a} L_{\omega} L_{\Omega}-L_{M \Omega} L_{\Omega a} L_{\omega}-\right. \\
& \left.\left.L_{M \omega}\left(L_{\omega a} L_{\Omega}-L_{\Omega a} L_{\omega \Omega}\right)\right) r, L_{M} L_{\omega} L_{\Omega} L_{a}\right] .
\end{aligned}
$$

From Eq. (8), it is easy to derive that $\left\{\mathscr{N}^{a}, \mathscr{N}^{\Omega}, \mathscr{N}^{\omega}, \mathscr{N}^{M}\right\}$ are integer numbers since there are the result of sums and multiplications of integer numbers. We can also derive that these new distribution variables can only present the values shown in the following expressions:

$$
\begin{aligned}
& \mathscr{N}^{a} \in\left\{1, \ldots, L_{a}\right\}, \\
& \mathscr{N}^{\Omega} \in \in\left\{1, \ldots, L_{\Omega} L_{a}\right\}, \\
& \mathscr{N}^{\omega} \in\left\{1, \ldots, L_{\omega} L_{\Omega} L_{a}\right\}, \\
& \mathscr{N}^{M} \in\left\{1, \ldots, L_{M} L_{\omega} L_{\Omega} L_{a}\right\},
\end{aligned}
$$

which define a set of possible values for each different dimension of the space. This means that we can define given values of the semi-major axis, the right ascension of the ascending node, the argument of perigee, and the initial mean anomaly for the satellites of the constellation to share. For instance, let $L_{\Omega} L_{a}=4$ be the number of different positions in the right ascension of the ascending node, that is, the number of different orbital planes of the constellation. If we relate $\mathscr{N}^{\Omega}=\{1,2,3,4\}$ to $\Delta \Omega_{1}=0^{\circ}, \Delta \Omega_{2}=30^{\circ}, \Delta \Omega_{3}=90^{\circ}$ and $\Delta \Omega_{4}=180^{\circ}$ respectively, we are setting the possible orbital planes in which the satellites of the constellation can be positioned. As it can be seen, it is not required to perform a uniform distribution in these variables.

\subsection{Complete uniform distribution}

In the previous subsection, we introduced a formulation to perform a uniform distribution over a set of predefined values of semi-major axis, right ascension of the ascending node, argument of perigee, and initial mean anomaly. However, no constraint was imposed in how these predefined values were distributed. Since complete uniform distributions are of importance for a large number of space missions, we deal with this particular case in the following lines. As said previously, the general formulation of 4DLFC leads to the expression:

$$
\begin{aligned}
\mathscr{N}_{i j k r}^{a}= & \bmod \left[r, L_{a}\right] \\
\mathscr{N}_{i j k r}^{\Omega}= & \bmod \left[L_{a} i-L_{\Omega a} r, L_{\Omega} L_{a}\right] \\
\mathcal{N}_{i j k r}^{\omega}= & \bmod \left[L_{\Omega} L_{a} k-L_{\omega \Omega} L_{a} i-\left(L_{\omega a} L_{\Omega}-L_{\omega \Omega} L_{\Omega a}\right) r, L_{\omega} L_{\Omega} L_{a}\right] \\
\mathscr{N}_{i j k r}^{M}= & \bmod \left[L_{\omega} L_{\Omega} L_{a} j-L_{M \omega} L_{\Omega} L_{a} k-\left(L_{M \Omega} L_{\omega} L_{a}-L_{M \omega} L_{\omega \Omega} L_{a}\right) i-\right. \\
& \left(L_{M a} L_{\omega} L_{\Omega}-L_{M \Omega} L_{\Omega a} L_{\omega}-\right. \\
& \left.\left.L_{M \omega}\left(L_{\omega a} L_{\Omega}-L_{\Omega a} L_{\omega \Omega}\right)\right) r, L_{M} L_{\omega} L_{\Omega} L_{a}\right]
\end{aligned}
$$


or in a more compact form:

$$
\left(\begin{array}{cccc}
L_{a} & 0 & 0 & 0 \\
L_{\Omega a} & L_{\Omega} & 0 & 0 \\
L_{\omega a} & L_{\omega \Omega} & L_{\omega} & 0 \\
L_{M a} & L_{M \Omega} & L_{M \omega} & L_{M}
\end{array}\right)\left(\begin{array}{c}
\mathscr{N}_{i j k r}^{a} \bmod \left(L_{a}\right) \\
\mathscr{N}_{i j k r}^{\Omega} \bmod \left(L_{\Omega} L_{a}\right) \\
\mathscr{N}_{i j k r}^{\omega} \bmod \left(L_{\omega} L_{\Omega} L_{a}\right) \\
\mathscr{N}_{i j k r}^{M} \bmod \left(L_{M} L_{\omega} L_{\Omega} L_{a}\right)
\end{array}\right)=\left(\begin{array}{c}
r \\
i \\
k \\
j
\end{array}\right)
$$

where $\left\{\mathscr{N}^{a}, \mathscr{N}^{\Omega}, \mathscr{N}^{\omega}, \mathscr{N}^{M}\right\}$ must be related with different values of the real distribution variables $a, \Omega, \omega$ and $M$ in order to obtain the final constellation distribution. In general, these values can be selected with no restriction. However, if a completely uniform distribution is required, the values of $\Omega, \omega$ and $M$ must be related with the distribution parameters of the problem in such a way that the possible values of these angles can present are uniformly distributed. In particular, and from Eq. (9):

$$
\begin{aligned}
\Delta \Omega_{i j k r} & =2 \pi \frac{\mathcal{N}_{i j k r}^{\Omega}}{L_{a} L_{\Omega}}, \\
\Delta \omega_{i j k r} & =2 \pi \frac{\mathscr{N}_{i j k r}^{\omega}}{L_{a} L_{\Omega} L_{\omega}}, \\
\Delta M_{i j k r} & =2 \pi \frac{\mathscr{N}_{i j k \nu}^{\mathscr{M}}}{L_{a} L_{\Omega} L_{\omega} L_{M}},
\end{aligned}
$$

where all these angles are related with one reference satellite $(0000)$ from the constellation. Note that this satellite distribution provides a configuration that is geometrically uniform in the same sense as it was presented in the 2DLFC and the 3D-LFC.

\subsection{Setting the values of the semi-major axes of the constellation}

Although the general methodology presented in the 4DLFC formulation allows to generate a large number of different distributions, in most applications it is extremely interesting that the structure of the constellation presents some periodic properties, and thus, additional constraints must be imposed in the semi-major axis in order to obtain that important characteristic.

Let $N_{p}$ be the number of complete orbits that a satellite requires in order to complete a closed track in a given frame of reference, and let $N_{d}$ be the number of complete rotations that the frame of reference performs during this time. For instance, if the selected frame of reference is the Earth Centered Earth Fixed (ECEF), $N_{p}$ and $N_{d}$ correspond to the minimum number of orbit revolutions and the minimum number of days that a satellite requires to repeat its ground-track. In that respect, it is worth noticing that even if an orbit does not repeat its ground-track in the ECEF frame of reference, it is possible to find another rotating frame of reference in which it does. However, in order to simplify the exposition and without loss of generality we will assume that the reference systems in consideration are the inertial and the ECEF frames of reference. Thus, we can define a period of repetition for each satellite as:

$$
T_{c}=N_{p} T=N_{d} T_{d}
$$

where $T$ is the orbital period of each satellite and $T_{d}$ is the rotating period of the reference frame. Additionally, $T_{c}$ is the shortest time for a satellite to repeat its dynamic in both reference systems. This means that for any time multiple of $T_{c}$ that is also true. In other words, let $T_{s}=A T_{c}$ where $A$ is a natural number, then after a time $T_{s}$ the satellite also repeats its dynamics in both reference frames.

Therefore, in order to obtain a periodic system, we have to find a time $T_{s}$ when all the satellites of the constellation repeat their dynamics at the same time. Let $N_{d}^{r}$ and $N_{p}^{r}$ be the number of orbital revolutions and the number of reference frame complete rotations that each subset of satellites with denomination $r$ need to perform in order to repeat their dynamic in both reference systems:

$T_{c}^{r}=N_{p}^{r} T^{r}=N_{d}^{r} T_{d}$

Then, and since the reference frames are the same for all the satellites of the constellation, $T_{d}$ is common for all the constellation. This implies that the time $T_{s}$ can be computed as follows:

$T_{s}=\operatorname{mcm}\left(N_{d}^{1}, N_{d}^{2}, \ldots, N_{d}^{r}, \ldots, N_{d}^{L_{a}}\right) T_{d}$,

where $\operatorname{mcm}\left(N_{d}^{1}, N_{d}^{2}, \ldots, N_{d}^{r}, \ldots, N_{d}^{L_{a}}\right)$ is the minimum common multiplier of the different $N_{d}^{r}$ of the constellation. On the other hand, the values of $N_{p}^{r}$ can be freely chosen, taking into account that their values will determine the semi-major axis of the orbits (remember that the period of the orbits $T^{r}$ is a function of the semi-major axis).

This means that for example, if we have a repeating ground-track constellation whose satellites have two different semi-major axes and that satellites repeat their ground-tracks in the same number of days, that leads to a dynamic that is repeated in $T_{s}=T_{c}=N_{d} T_{d}$ (since $\left.\operatorname{mcm}\left(N_{d}^{1}, N_{d}^{2}\right)=N_{d}\right)$. If instead we have two different repetition periods, $T_{s}=\operatorname{mcm}\left(N_{d}^{1}, N_{d}^{2}\right) T_{d}$.

\section{Including the $J 2$ perturbation in the formulation}

The former formulation considers a Keplerian movement where satellites are only subjected to the main term of the gravitational potential of the primary body. Another possible application is in missions where part of the fuel budget is dedicated to maintain the different dynamics of the satellites in the constellation within a given mission boundary. However, there are space missions where it is of interest to include the oblateness effect of the Earth $\left(\mathrm{J}_{2}\right.$ of the gravitational potential) in the design process of the constellation due to the important effects that this perturbation produces. In particular, the $J_{2}$ perturbation generates a shifting of the orbital planes and a rotation of the orbits inside their planes. This causes the destruction of the initial design of the constellation if no orbital maneuvers are performed, being the effect more noticeable the closer satellites are from Earth. For these reasons, we include 
in the constellation design proposed the effects of the $J_{2}$ perturbation.

The secular variations of the classical elements for a satellite orbiting the Earth under the $J_{2}$ perturbation are (Vallado, 2001):

$$
\begin{array}{ll}
\dot{a}_{s e c}=0 ; & n=\sqrt{\frac{\mu}{a^{3}}}\left[1+\frac{3}{4} J_{2}\left(\frac{R_{\oplus}}{a\left(1-e^{2}\right)}\right)^{2}\left(2-3 \sin ^{2}(\text { inc })\right) \sqrt{1-e^{2}}\right] ; \\
\dot{e}_{s e c}=0 ; & \dot{\omega}_{s e c}=\frac{3}{4} J_{2}\left(\frac{R_{\oplus}}{a\left(1-e^{2}\right)}\right)^{2} n\left(5 \cos ^{2}(i n c)-1\right) ; \\
\dot{i}_{s e c}=0 ; & \dot{\Omega}_{s e c}=-\frac{3}{2} J_{2}\left(\frac{R_{\oplus}}{a\left(1-e^{2}\right)}\right)^{2} n \cos (i n c) ;
\end{array}
$$

where $\mu$ and $R_{\oplus}$ are the gravitational constant and the equatorial radius of the Earth respectively, and $n$ is the mean motion of the satellite. Thus, there are three secular variables that change over time: the right ascension of the ascending node, the argument of perigee and the mean motion. In order to maintain the configuration as a constellation, we require that all the orbital planes shift at the same speed $\left(\dot{\Omega}_{s e c}=\dot{\Omega}_{s e c 0}\right)$, that the rotations in the orbital plane are performed at the same speed $\left(\dot{\omega}_{s e c}=\dot{\omega}_{s e c}\right)$, and that the period of the orbits is congruent with the repetition period of the whole structure $\left(T_{s}\right.$ must be the same for all the satellites of the constellations), that is, there must exist a time period when the constellation repeats its dynamic. We denote $\dot{\Omega}_{s e c 0}$ and $\dot{\omega}_{\sec 0}$ to the constellation references for the secular variations of the right ascension of the ascending node and the argument of perigee. On the other hand, and for a given satellite of the constellation, there exists a relation between the different nodal periods:

$T_{c}=N_{p} T_{\Omega}=N_{p} \frac{2 \pi}{n+\dot{\omega}}=N_{d} T_{\Omega G}=N_{d} \frac{2 \pi}{\omega_{\oplus}-\dot{\Omega}}$,

where $T_{\Omega}$ is the nodal period of the orbit, and $T_{\Omega G}$ is the nodal period of Greenwich. Thus, using Eq. (16) and the former constraints, we obtain:

$$
\begin{aligned}
& \frac{2 \pi}{T_{\Omega G}} \frac{N_{p}}{N_{d}}=\sqrt{\frac{\mu}{a^{3}}}\left[1+\frac{3}{4} J_{2}\left(\frac{R_{\oplus}}{a\left(1-e^{2}\right)}\right)^{2}\left(2-3 \sin ^{2}(i n c)\right) \sqrt{1-e^{2}}\right]+\dot{\omega}_{\text {sec } 0}, \\
& \dot{\omega}_{\text {sec } 0}=\frac{3}{4} J_{2}\left(\frac{R_{\oplus}}{a\left(1-e^{2}\right)}\right)^{2} \frac{2 \pi}{T_{\Omega G}} \frac{N_{p}}{N_{d}}\left(5 \cos ^{2}(i n c)-1\right), \\
& \dot{\Omega}_{\text {sec } 0}=-\frac{3}{2} J_{2}\left(\frac{R_{\oplus}}{a\left(1-e^{2}\right)}\right)^{2} \frac{2 \pi}{T_{\Omega G}} \frac{N_{p}}{N_{d}} \cos (\text { inc }),
\end{aligned}
$$

which is a system of three nonlinear equations with three unknowns (the semi-major axis, the inclination and the eccentricity) for each different value of $\mathscr{N}^{a}$. From the expressions of $\dot{\omega}_{\sec 0}$ and $\dot{\Omega}_{s e c 0}$, we can derive that:

$$
\cos (i n c)=\frac{-\dot{\omega}_{s e c 0} \pm \sqrt{\dot{\omega}_{s e c 0}^{2}+5 \dot{\Omega}_{s e c 0}^{2}}}{5 \dot{\Omega}_{s e c 0}},
$$

meaning that all the satellites in the constellation must have the same inclination (the one of the reference orbit), since $\dot{\omega}_{s e c 0}$ and $\dot{\Omega}_{s e c 0}$ are common for the constellation. On the other hand, from the expression in $\dot{\Omega}_{s e c 0}$ we obtain a relation between the semi-major axis and the eccentricity of the orbit:
$1-e^{2}=\frac{R_{\oplus}}{a} \sqrt{-\frac{3}{2} \frac{J_{2}}{\dot{\Omega}_{\text {sec } 0}} \frac{2 \pi}{T_{\Omega G}} \frac{N_{p}}{N_{d}} \cos (\text { inc })}$,

which can be introduced in the expression of the mean motion, obtaining a nonlinear equation in the semi-major axis:

$$
\begin{aligned}
a^{\frac{3}{2}}= & \left(\frac{2 \pi}{T_{\Omega G}} \frac{N_{p}}{N_{d}}-\dot{\omega}_{\text {seco }}\right)^{-1} \\
& \times \sqrt{\mu}\left[1-\frac{\dot{\Omega}_{\text {seco }}}{4 \pi} \frac{T_{d} N_{d}}{N_{p}} \frac{2-3 \sin ^{2}(i n c)}{\cos (i n c)} \sqrt{\frac{R_{\oplus}}{a} \sqrt{-\frac{3}{2} \frac{J_{2}}{\dot{\Omega}_{s e c 0}} \frac{2 \pi}{T_{\Omega G}} \frac{N_{p}}{N_{d}} \cos (i n c)}}\right],
\end{aligned}
$$

from where the value of the semi-major axis can be obtained numerically, and then, the value of the eccentricity using Eq. (20).

This means that all satellites of the constellation that share the same value of $\mathscr{N}^{a}$ have the same values of semi-major axis, inclination and eccentricity. This is effectively generating a 3D Lattice Flower Constellation inside the 4D configuration. Thus, a 4D Lattice Flower Constellation can also be regarded as a set of 3D Lattice Flower Constellations that present a given relation in their motions provided by Eq. (15).

The previous conditions can be relaxed for some missions. For instance, if we are interested in designing a sun-synchronous constellation, it is possible to only impose that $\dot{\Omega}_{s e c}=\dot{\Omega}_{s e c 0}$ and select the sun-synchronous inclination for each altitude. This means that the constellation will not be coordinated in $n$ and $\omega$; but, it will maintain the orbital plane structure over time. In that sense, other design combinations are also possible.

Finally, it is important to note that under additional perturbations, the satellites of the constellation will require to perform orbital maneuvers in order to maintain the configuration defined (Arnas, 2018; Arnas et al., 2016). This is more important in a 4D Lattice Flower Constellation since eccentricity and semi-major axis are in general different for each satellite of the constellation.

\section{Relation with 2D and 3D Lattice Flower Constellations}

4D-LFC contains as a subset the former 2D-LFC and 3D-LFC. In order to prove that, we must depart from the complete uniform distribution presented in Section 3.3 and impose that the distribution only has one semi-major axis that is shared for all the satellites of the constellation, that is, we must impose that $L_{a}=1$. That way, by introducing these constraints in Eq. (8), we obtain:

$$
\begin{aligned}
\mathcal{N}_{i j k r}^{a} & =1, \\
\Delta \Omega_{i j k r} & =2 \pi \frac{\bmod \left[i, L_{\Omega}\right]}{L_{\Omega}}, \\
\Delta \omega_{i j k r} & =2 \pi \frac{\bmod \left[L_{\Omega} k-L_{\omega \Omega} i, L_{\omega} L_{\Omega}\right]}{L_{\Omega} L_{\omega}}, \\
\Delta M_{i j k r} & =2 \pi \frac{\bmod \left[L_{\omega} L_{\Omega} j-L_{M \omega} L_{\Omega} k-\left(L_{M \Omega} L_{\omega \omega}-L_{M \omega} L_{\omega \Omega}\right) i L_{M} L_{\omega} L_{\Omega}\right]}{L_{\Omega} L_{\omega} L_{M}},
\end{aligned}
$$


which can be operated to obtain a simpler expression if we take into account that angles are module $2 \pi$ :

$$
\begin{aligned}
\mathscr{N}_{i j k r}^{a} & =1, \\
\Delta \Omega_{i j k r} & =2 \pi \frac{i}{L_{\Omega}}, \\
\Delta \omega_{i j k r} & =2 \pi\left(\frac{k}{L_{\omega}}-\frac{L_{\omega \Omega} i}{L_{\Omega} L_{\omega}}\right), \\
\Delta M_{i j k r} & =2 \pi\left(\frac{j}{L_{M}}-\frac{L_{M \omega} k}{L_{\omega} L_{M}}-\frac{\left(L_{M \Omega} L_{\omega}-L_{M \omega} L_{\omega \Omega}\right) i}{L_{\Omega} L_{\omega} L_{M}}\right),
\end{aligned}
$$

which is the same expression seen in Eq. (2) for the 3DLFC but with the notation introduced in the 4D-LFC. A similar relation can be established with $2 \mathrm{D}-\mathrm{LFC}$ by imposing in addition that $L_{\omega}=1$, that way:

$$
\begin{aligned}
\mathscr{N}_{i j k r}^{a} & =1, \\
\Delta \Omega_{i j k r} & =2 \pi \frac{i}{L_{\Omega}}, \\
\Delta \omega_{i j k r} & =0, \\
\Delta M_{i j k r} & =2 \pi\left(\frac{j}{L_{M}}-\frac{L_{M \Omega} i}{L_{\Omega} L_{M}}\right),
\end{aligned}
$$

which represents the same distribution as the one seen in Eq. (1) for 2D-LFC. This means that, 4D-LFC represent the mathematical generalization of the former 2D-LFC and 3D-LFC.

Additionally, if instead of limiting the number of semimajor axes of the constellation to $L_{a}=1$ we study a general distribution where $L_{a} \neq 1$, it is also possible to observe 2DLFC and 3D-LFC inside the resultant distribution. Let $r$ be the distribution variable that defines a given value of semimajor axis. Then, we can study all the satellites that share this semi-major axis and select one of them as the reference satellite for this subset. That way, we can relate the positions of this subset of satellites with respect to this reference satellite using Eq. (8):

$$
\begin{array}{ll}
\mathscr{N}_{i j k r}^{a} & =r, \\
\Omega_{i j k r}-\Omega_{000 r} & =2 \pi \frac{i}{L_{\Omega}}, \\
\omega_{i j k r}-\omega_{000 r} & =2 \pi\left(\frac{k}{L_{\omega}}-\frac{L_{\omega \Omega \Omega} i}{L_{\Omega} L_{\omega}}\right), \\
M_{i j k r}-M_{000 r} & =2 \pi\left(\frac{j}{L_{M}}-\frac{L_{M \omega} k}{L_{\omega} L_{M}}-\frac{\left(L_{M \Omega} L_{\omega}-L_{M \omega} L_{\omega \Omega}\right) i}{L_{\Omega} L_{\omega} L_{M}}\right),
\end{array}
$$

which it is again a $3 \mathrm{D}-\mathrm{LFC}$ distribution but with respect to the reference satellite that we have selected at this semimajor axis. Therefore, we can conclude that $4 \mathrm{D}-\mathrm{LFC}$ is in fact a combination of 2D-LFC and 3D-LFC into a larger constellation that presents the properties of uniformity, symmetry and periodicity in its configuration.

\section{Simple example of application}

In this section an example of $4 \mathrm{D}-\mathrm{LFC}$ is presented to show the possibilities of this methodology. In particular, we propose a satellite constellation based on two subsets of satellites located at different altitudes $\left(L_{a}=2\right)$. The first subset corresponds to deep space observation satellites, which will be located at the higher orbit. It is assumed that these satellites are not able to establish a correct communication with their ground stations, and thus they require the assistance of other satellites to accomplish this task. On the other hand, the second subset is comprised by telecommunication satellites whose mission is to receive the data sent by the first subset and resend it to their ground stations.

In order to present a clear example, we consider a constellation based on 10 repeating ground track satellites whose period of repetition is 1 day, that is, $N_{d}^{1}=N_{d}^{2}=1$, and that are all contained in the same orbital plane. In particular, we denote with $r=1$ to the 5 telecommunication satellites, which are located in the lower orbit; and with $r=2$ to the 5 deep space observation satellites, which are positioned in the higher orbit. Note that with this distribution the constellation has $L_{M}=5$ satellites per orbit. These satellites will repeat their orbital motion in $N_{p}^{1}=5$ and $N_{p}^{2}=4$ orbital revolutions respectively. Moreover, having all satellites positioned in the same orbital plane implies that $L_{\Omega}=1$. Finally, a circular orbit at the critical inclination $\left(i n c=63.56^{\circ}\right)$ is considered for the telecommunication satellites. Finally, we can consider without loss of generality that $L_{\omega}=1$.

Now, there are two possibilities in order to perform the nominal design of the constellation. The first one is based on generating two circular orbits for the constellation where it will be required to compensate the different drift of the orbits by orbital maneuvers. The second possibility is to define a nominal constellation with the formulation presented in Section 4. In the next sections we present both approaches to this example of constellation design.

\subsection{Design based on circular orbits}

The idea of this design is to generate two circular orbits, one at a higher altitude where the deep space observation satellites will be located, and another at a lower altitude containing the telecommunication satellites of the constellation. That way, using Eqs. (17) and (18) the values of the two different semi-major axes presented in the constellation can be obtained: $a_{1}=14419.333 \mathrm{~km}$ and $a_{2}=16732.336 \mathrm{~km}$, which are related to $\mathscr{N}_{i j k 1}^{a}=1$ and $\mathscr{N}_{i j k 2}^{a}=2$ respectively. Then, and since the distribution is only performed in the semi-major axis and the mean anomaly of the satellites (argument of perigee and right ascension of the ascending node are common for the constellation), Eq. (8) leads to:

$$
\begin{aligned}
\mathscr{N}_{i j k r}^{a} & =r, \\
\Delta \Omega_{i j k r} & =0, \\
\Delta \omega_{i j k r} & =0, \\
\Delta M_{i j k r} & =2 \pi\left(\frac{j}{L_{M}}-\frac{L_{M a} r}{L_{M} L_{a}}\right),
\end{aligned}
$$

where $L_{M a}=\{0,1\}$ is the combination number, a parameter that allows to shift the satellite distribution between both orbits. In particular, the only two configurations that can be obtained using the 4D-LFC formulation are presented in Figs. 3 and 4 respectively. Note that the selection 


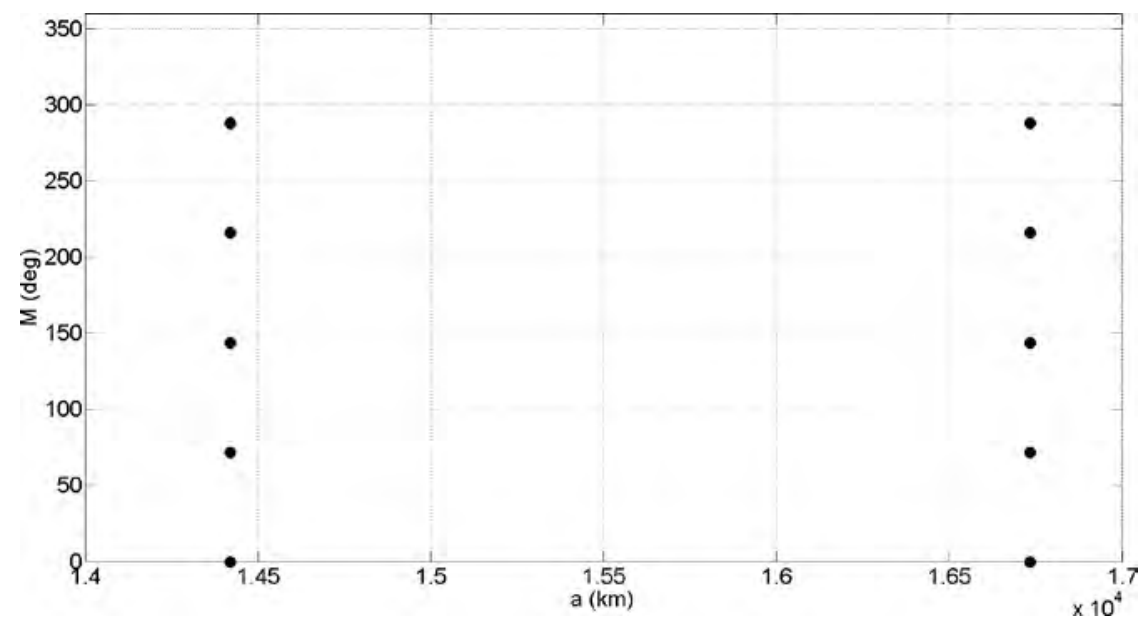

Fig. 3. Satellite configuration with $L_{M a}=0$.

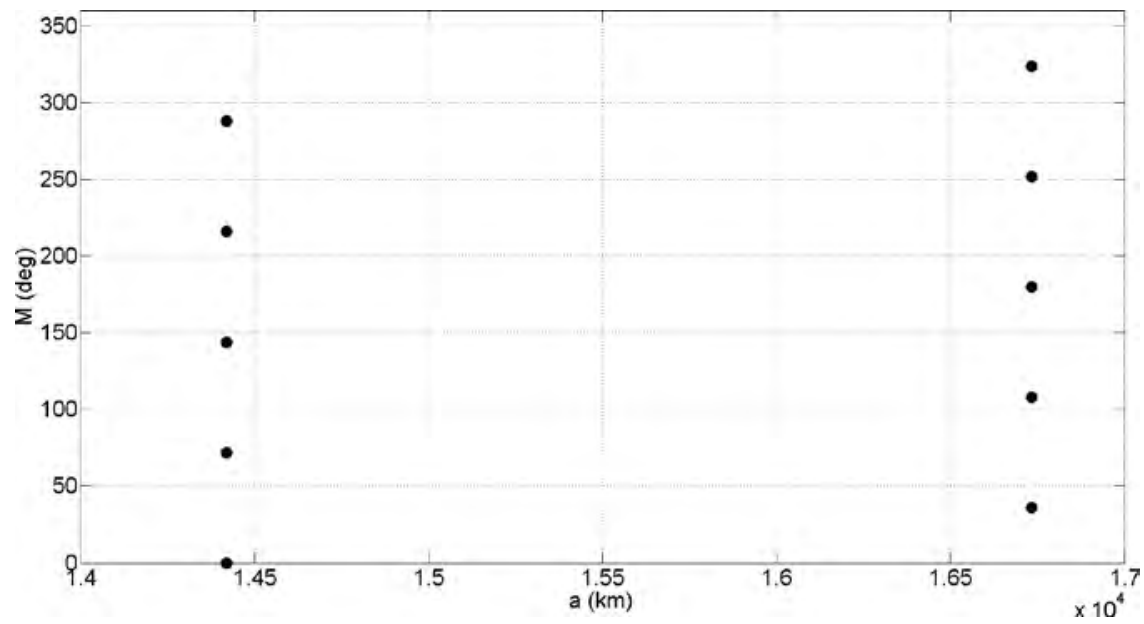

Fig. 4. Satellite configuration with $L_{M a}=1$.

of one of these configurations will determine the latitude above the Earth where the communication between satellites can be established.

Then, and from the distributions presented in Figs. 3 and 4 we are able to select one configuration. For the purpose of this example, the distribution related with $L_{M a}=0$ is chosen as the configuration of the constellation. Using that satellite distribution, the dynamics of the whole constellation can be computed. In that respect, a graphical representation of the inertial orbits of the constellation has been included in Fig. 5.

This distribution allows that given a pair of satellites (one from the higher orbit and the other from the lower orbit), a communication link between them can be established once per day since this is the number of times that the satellite in the lower orbit overtakes the other in one day. This means that each satellite from the higher orbit has the opportunity to communicate five times a day with the assistance of the satellites in the lower orbit.
Finally, it is interesting to study the propellant that the satellites of this constellation require in order to maintain the dynamic between the two subsets of satellites under the effect of the $J_{2}$ perturbation. In that regard, it is assumed that, as a mission requirement, the orbital planes between both subsets of satellites cannot be farther than $1.5^{\circ}$, that is, $\Delta \Omega_{\max }=1.5^{\circ}$. This constraint allows to define a maximum relative variation of the distribution from its nominal definition. This orbit maintenance is performed using impulsive orbital maneuvers, and in order to distribute the maintenance effort between all the satellites of the constellation, we impose that all satellites have to spend the same $\Delta v$ during this maneuvers, that is, all the satellites have to correct their orbits with respect to a reference secular variation in $\Omega$ defined for the whole constellation. That way, a maneuver to compensate the differential drift of the orbital plane must be performed each 28 days, requiring an impulse of $\Delta v=130.151 \mathrm{~m} / \mathrm{s}$ per maneuver in each satellite of the constellation. 


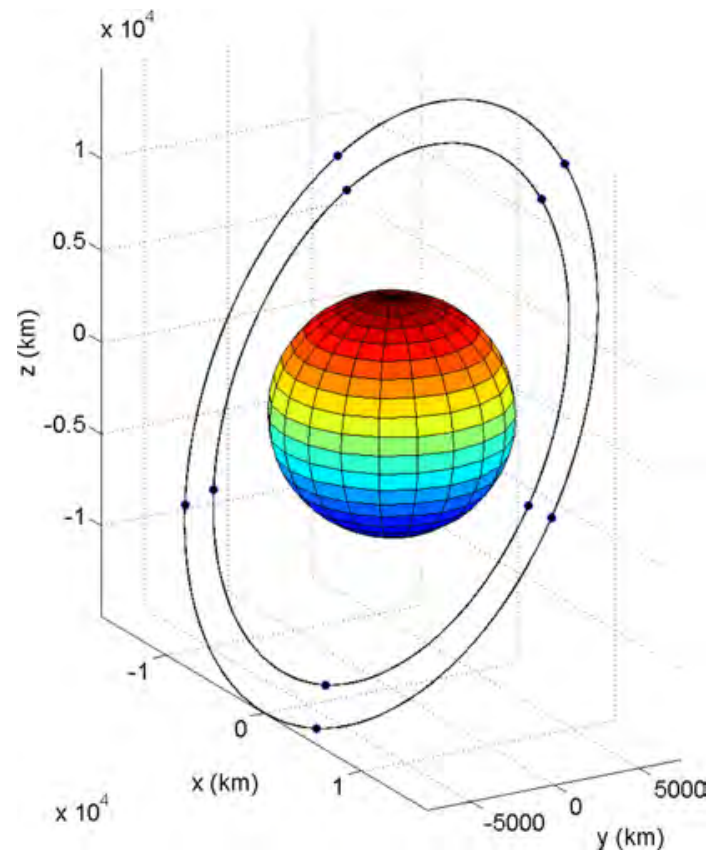

Fig. 5. Inertial representation of the constellation based on circular orbits.

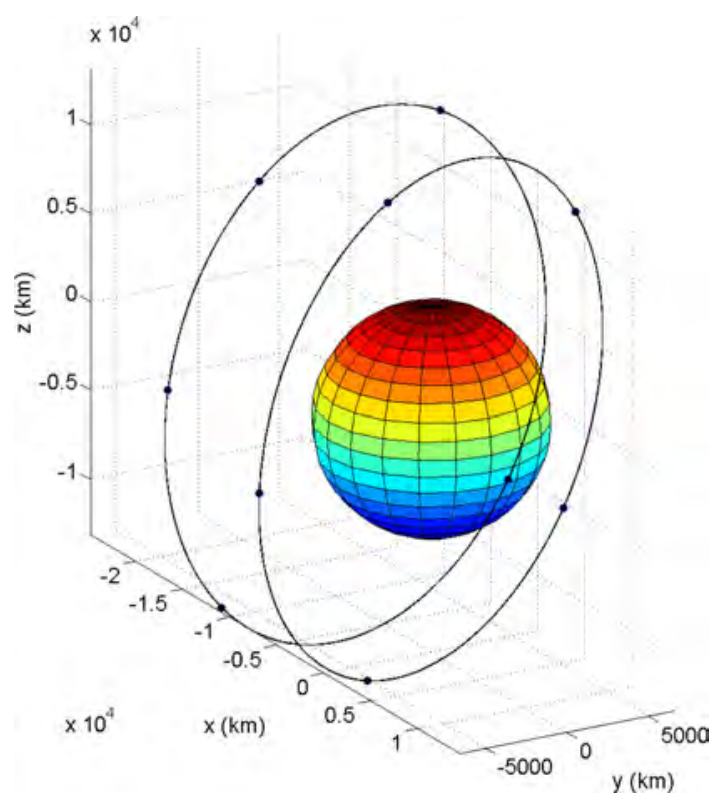

Fig. 6. Inertial representation of the constellation based on $J_{2}$ coordination.

\subsection{Design based on $J_{2}$ coordination}

In this case, the same basic example is studied but using the $J_{2}$ coordinated dynamic shown in Section 4. That way, a constellation consisting on a circular orbit at a lower semi-major axis (containing the telecommunication satellites) and an eccentric orbit at a higher semi-major axis (where the deep space observation satellites are located) is generated. In particular, using Eqs. (20) and (21), the semi-major axes of the constellation are now $a_{1}=14419.944 \mathrm{~km}$ and $a_{2}=16732.085 \mathrm{~km}$, while their eccentricities are $e_{1}=0.0$ and $e_{2}=0.479$.

As in the former example, the distribution can be defined using Eq. (26) where again two possibilities of distribution can be generated: $L_{M a}=\{0,1\}$. In this case, $L_{M a}=0$ is chosen since it provides a more beneficial dynamics and geometry for this problem. Therefore, the distribution follows an equivalent distribution to the one presented in Fig. 3. A graphical representation of the constellation orbits can be seen in Fig. 6. As it can be observed, both orbits have an intersection. This could, in general, produce a collision avoidance problem for the constellation. However, this problem has been solved by maximizing the distances between satellites when passing that intersection point. This was done by selecting a proper value of the configuration number $L_{M a}$.

Regarding the possibilities of communication between both subsets of satellites, we assume that this is only possible when the deep space observation satellites fly above the telecommunication satellites, that is, when these satellites are near their apogee. If that is the case, each deep space observation satellite is able to communicate a minimum of two times per day with the telecommunication satellites, and a maximum of three times per day. If instead, the communication can be established at any point during the dynamic of the system, each deep space observation satellite can perform this communication five times per day, the same amount than the former example.

Finally, it is also possible to study the fuel budget required to maintain this dynamic under the effect of the $J_{2}$ perturbation. However, since the effects of the $J_{2}$ perturbation are already included in the nominal definition of the constellation, both orbits shift at the same rate, and thus, no additional maneuvers are required to compensate this effect. In particular, note that the secular values of the orbital elements of all the satellites of this constellation fulfill $\dot{\Omega}_{s e c}=\dot{\Omega}_{s e c 0}, \dot{\omega}_{s e c}=\dot{\omega}_{\sec 0}$ and Eq. (17), and thus, the secular relative distribution of the constellation is maintained under the $J_{2}$ perturbation.

\section{Example of application to mega-constellations}

This example will focus on the design of a megaconstellation, that is, a constellation of satellites comprised by a large number of spacecrafts. In that respect, we propose to uniformly distribute 5000 Earth observation satellites at 5 different altitudes between $650 \mathrm{~km}$ and $850 \mathrm{~km}$ using the design proposed in this manuscript. In particular, and since the objective is Earth observation, the orbits of the constellation will be sun-synchronous and circular. Moreover, we will also assume that no coordination is performed between satellites at different altitudes in order to maintain the sun-synchronous property of every orbit no matter the altitude in which the spacecraft is located. 
In general, one important property that megaconstellations have to present is that no satellite conjunction should appear in the configuration during the nominal operation of spacecrafts. This allows to drastically reduce the probability of collision between satellites of the constellation despite the spacecraft density. Therefore, the design proposed in this example will focus on maximizing the minimum distances between spacecrafts. In order to ease this computation, this work makes use of the expression provided by Speckman et al. (1990):

$\rho_{\min }=2\left|\sqrt{\frac{1+\cos ^{2}(\text { inc })+\sin ^{2}(\text { inc }) \cos (\Delta \Omega)}{2}} \sin \left(\frac{\Delta F}{2}\right)\right|$,

where:

$\Delta F=\Delta M-2 \tan ^{-1}\left[-\cos (\right.$ inc $\left.) \tan \left(\frac{\Delta \Omega}{2}\right)\right]$,

which relates the minimum dimensionless distance $\left(\rho_{\min }=d_{\min } / a\right)$ between two satellites flying in circular orbits at the same altitude and inclination (inc), with their difference in right ascension of the ascending node $(\Delta \Omega)$ and mean anomaly $(\Delta M)$.

From the initial conditions of this example, $L_{a}=5(5$ different altitudes), $L_{\omega}=1$ and $L_{\omega a}=L_{\omega \Omega}=L_{M \omega}=0$ (orbits are circular), and $L_{a} L_{\Omega} L_{\omega} L_{M}=5000$ (the constellation has exactly 5000 satellites). Note that this also implies that $L_{\Omega} L_{M}=1000$. That way, the satellite distribution can be defined as:

$$
\begin{aligned}
a_{i j k r} & =R_{\oplus}+600+50 r \quad(\mathrm{~km}), \\
\Delta \Omega_{i j k r} & =2 \pi\left(\frac{i}{L_{\Omega}}-\frac{L_{\Omega a}}{L_{\Omega}} \frac{r}{L_{a}}\right), \\
\Delta \omega_{i j k r} & =0 \\
\Delta M_{i j k r} & =2 \pi\left(\frac{j}{L_{M}}-\frac{L_{M \Omega}}{L_{M}} \frac{i}{L_{\Omega}}-\frac{r}{L_{a}}\left(\frac{L_{M a}}{L_{M}}-\frac{L_{M \Omega}}{L_{M}} \frac{L_{\Omega a}}{L_{\Omega}}\right)\right),
\end{aligned}
$$

where we can realize that if the distribution variable $r$ is fixed, this expression defines a 2D-LFC as seen in Section 5. Therefore, it is possible to reduce the searching space of solutions. In particular, the only free distribution parameters affecting the minimum distance between satellites are $L_{\Omega}, L_{M}$, and $L_{M \Omega}$; while $L_{\Omega a}$, and $L_{M a}$ only change the relative phasing of orbital planes and satellite locations between orbits at different altitudes.

That way, an exhaustive search of 4D-LFCs has been performed by varying the distribution parameters $L_{\Omega}, L_{M}$, and $L_{M \Omega}$, and taking into account that $L_{\Omega} L_{M}=1000$ and $L_{M \Omega} \in\left\{0, \ldots, L_{\Omega}-1\right\}$. The result of this search shows that constellations with $L_{\Omega}=500, L_{M}=2$, and $L_{M \Omega}=497$ present the overall larger minimum distance between satellites under the conditions considered. Fig. 7 shows the $(\Omega, M)$ space representation of the constellation for a given altitude. As it can be seen, the distribution is uniform in this projected representation, having each orbit (vertical lines) two satellites from the constellation. Moreover, Table 1 shows a summary of the minimum distances $\left(d_{\min }\right)$, and minimum angular distances $\left(\alpha_{\min }\right)$, computed as a function of the altitude of the orbits.

Once $L_{\Omega}, L_{M}$, and $L_{M \Omega}$ have been selected, $L_{\Omega a}$, and $L_{M a}$ remain as free parameters to choose for this problem. Particularly, if we want that no orbital plane is shared by satellites from different altitudes, for instance to prevent telecommunication or observation interference between satellites, then $L_{\Omega a}$ has to be selected such that $\operatorname{gcd}\left(L_{\Omega}, L_{\Omega a}\right)=1$, for example $L_{\Omega a}=2$. On the other hand, $L_{M a}$ controls the phasing of satellites with respect to the chosen altitude. Since no coordination on the orbital periods was imposed, this distribution parameter will only control the sequence in which satellites from different altitudes

Table 1

Minimum distances between satellites.

\begin{tabular}{llll}
\hline Altitude $(\mathrm{km})$ & Inc. $(\mathrm{deg})$ & $\alpha_{\min }(\mathrm{deg})$ & $d_{\min }(\mathrm{km})$ \\
\hline 650 & 97.980 & 0.980 & 120.204 \\
700 & 98.188 & 0.977 & 120.748 \\
750 & 98.393 & 0.975 & 121.283 \\
800 & 98.603 & 0.972 & 121.808 \\
850 & 98.816 & 0.970 & 122.322 \\
\hline
\end{tabular}

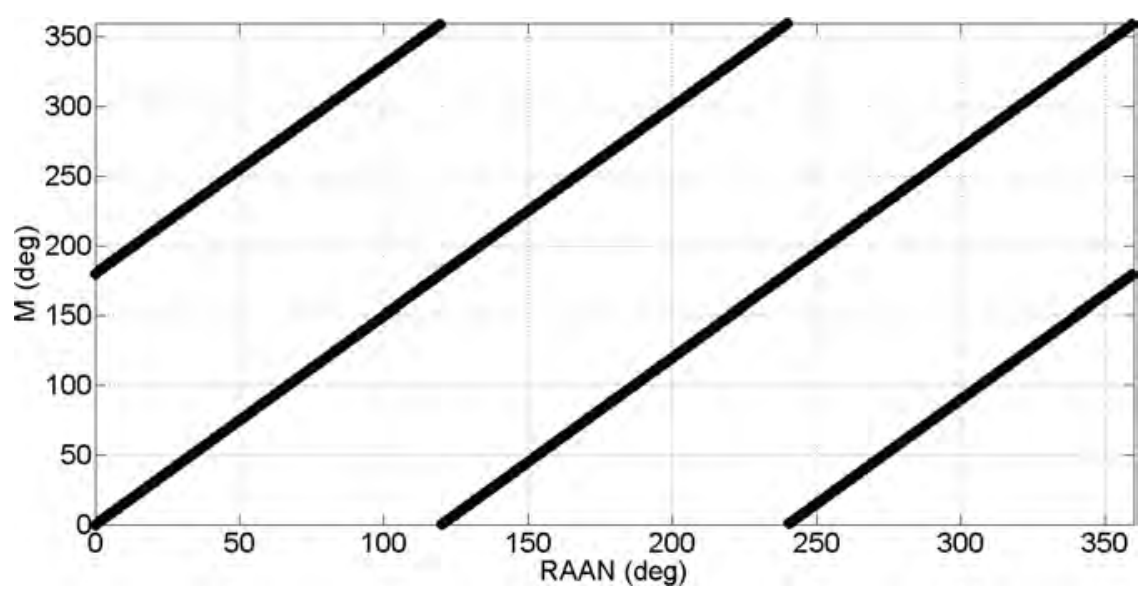

Fig. 7. $(\Omega, M)$-space representation of one altitude from the constellation. 


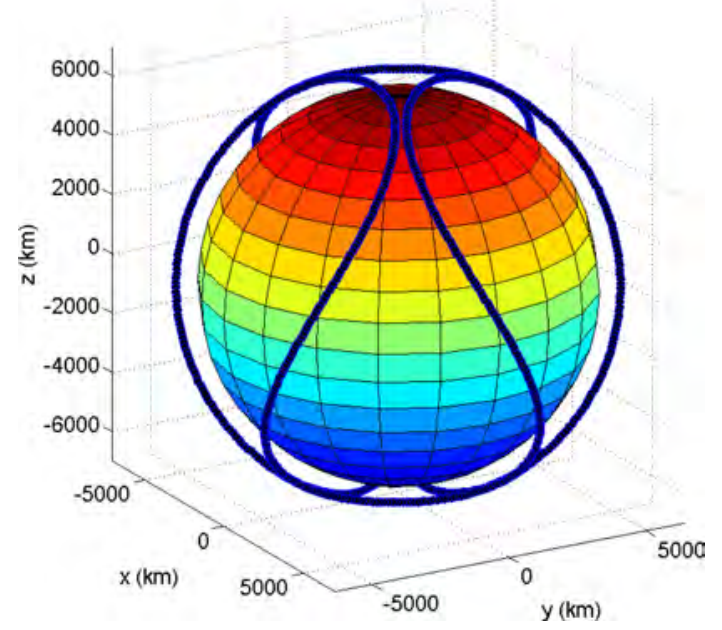

Fig. 8. Inertial representation of one altitude of the constellation.

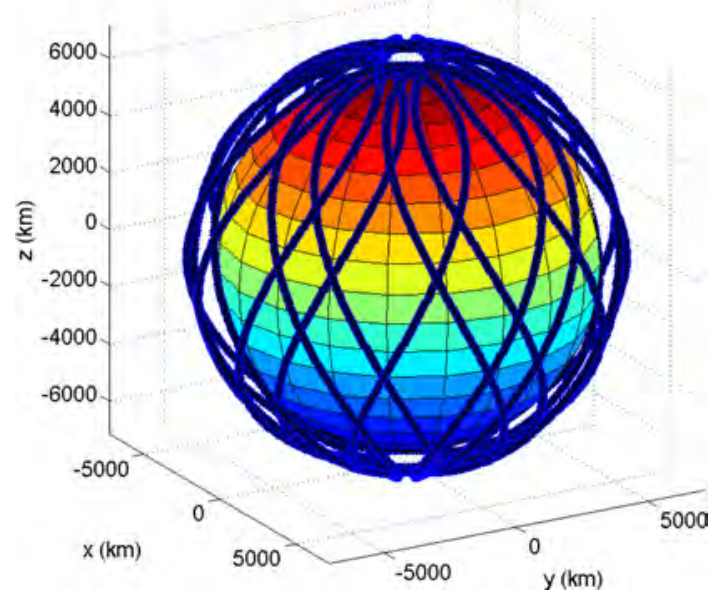

Fig. 9. Inertial representation of the whole mega-constellation.

will fly over Earth's Equator. Therefore, in this example we choose $L_{M a}=0$ for simplicity.

Fig. 8 shows the constellation distribution of one of the altitudes of the constellation. In here, it can be seen that the satellites of the constellation form a closed line that has no self-intersections, which in effect prevents the appearance of conjunctions in the dynamics as was discussed in Lee et al. (2015). On the other hand, Fig. 9 presents the whole constellation. As it can be seen, the selection of the distribution parameter $L_{\Omega a}=2$ has allowed to distribute this closed lines made of satellites evenly in the space, or in other words, satellites are better distributed around the Earth.

Note also that the results in number of satellites presented in this example are within the capacity limits shown in Arnas et al. (2020) for uniform sun-synchronous constellations. This means that if we increase the number of satellites in the configuration or allow non uniform distributions, it is still possible to find constellations with more spacecrafts but that still maintain the same minimum separation between satellites.

\section{Conclusion}

This work introduces the 4D Lattice Flower Constellations as a new framework in satellite constellation design. This methodology is based on the idea of generating constellations whose satellites are located at different semimajor axes while maintaining a periodic dynamic of the whole system. That way it is possible to define constellations whose satellites have very different instruments (and thus different mission requirements) or to coordinate the dynamic of different satellite constellations. This situations can arise, for instance, in telecommunications, or in the interference problem between constellations at a different altitudes.4D Lattice Flower Constellations is a design methodology that allows to generate all the possible constellations that are completely uniform with a given number of satellites. This is based on the idea of generating a lattice (a uniform distribution of points) in a four dimensional space. This concept was already applied in the 2D and 3D Lattice Flower Constellations, obtaining good results for global coverage missions, telecommunication constellations and global positioning systems. In here, the 4D Lattice Flower Constellations are introduced as a methodology to coordinate different constellations while maintaining the characteristic properties of uniformity, symmetry and periodicity of previous Lattice Flower Constellations.

Additionally, 4D Lattice Flower Constellations can be regarded as the mathematical generalization of the former 2D and 3D Lattice Flower Constellations. In fact, 2D and 3D Lattice Flower Constellations can be defined as a particular case of application of 4D Lattice Flower Constellations. In that respect, it is also interesting to note that inside the distribution of a 4D Lattice Flower Constellation, it is possible to observe complete $2 \mathrm{D}$ and $3 \mathrm{D}$ Lattice Flower Constellations generated by a subset of constellation satellites. This shows that 4D Lattice Flower Constellations can be used as a tool to coordinate the dynamic of several Lattice Flower Constellations.

Moreover, this manuscript includes the study of the effects of the $J_{2}$ perturbation on 4D Lattice Flower Constellations when a coordinated dynamic between satellites at different altitudes is required. In particular, we present a methodology to include the effects of this perturbation on the nominal design of the constellation. That way, it is possible to impose that all the orbits of the constellation shift at the same rate, which allows to maintain the relative configuration of the constellation under this perturbation with no additional orbital maneuvers. This methodology of design allows to reduce the fuel required to maintain the orbits, however, it limits the freedom of design. For that reason, this work also includes the study of the fuel budget required for the constellation when no natural coordination with $J_{2}$ is performed. 
Finally, it is also important to note that the Necklace Theory of Flower Constellations can also be applied to the 4D Lattice Flower Constellations. This will provide a wider range of possibilities of design for this methodology. It will also allow, for example, to study the problem of determining the optimal launching sequence for a constellation in such a way that the incomplete configuration presents functionality.

\section{Acknowledgments}

The work of David Arnas, Daniel Casanova, and Eva Tresaco was partially supported by the Spanish Ministry of Economy and Competitiveness, Project No. ESP201787113-R (AEI/FEDER, UE), and by the Aragon Government and European Social Fund (group E24_17R).

\section{References}

Arnas, D., 2018. Necklace Flower Constellations. Ph.D. thesis. Universidad de Zaragoza.

Arnas, D., Casanova, D., Tresaco, E., 2016. Relative and absolute stationkeeping for two-dimensional-lattice flower constellations. J. Guidance, Control, Dynam., 2602-2604 https://doi.org/10.2514/1.G000358.

Arnas, D., Casanova, D., Tresaco, E., 2017a. 2D Necklace Flower Constellations. Acta Astronaut. 142, 18-28. https://doi.org/10.1016/j. actaastro.2017.10.017.

Arnas, D., Casanova, D., Tresaco, E., 2017b. Time distributions in satellite constellation design. Celestial Mech. Dynam. Astron. 128 (23), 197-219. https://doi.org/10.1007/s10569-016-9747-3.

Arnas, D., Casanova, D., Tresaco, E., Mortari, D., 2017c. 3-Dimensional Necklace Flower Constellations. Celestial Mech. Dynam. Astron. 129 (4), 433-448. https://doi.org/10.1007/s10569-017-9789-1.

Arnas, D., Casanova, D., Tresaco, E., Mortari, D., 2017d. 3D Lattice Flower Constellations using necklaces. Adv. Astronaut. Sci. 160, 1681-1700.

Arnas, D., Lifson, M., Linares, R., Avendaño, M., 2020. Low earth orbit slotting for space traffic management using flower constellation theory. In: AIAA Scitech 2020 Forum. p. 0721. doi:10.2514/6.2020-0721.

Avendaño, M., Mortari, D., 2009. Rotating symmetries in space: the flower constellations. In: AAS 09-189, AAS/AIAA Space Flight Mechanics Meeting Conference. pp. 09-189.

Avendaño, M.E., Davis, J.J., Mortari, D., 2013. The 2-D lattice theory of Flower Constellations. Celestial Mech. Dynam. Astron. 116 (4), 325337. https://doi.org/10.1007/s10569-013-9493-8.
Casanova, D., Avendaño, M., Mortari, D., 2014a. Seeking gdop-optimal flower constellations for global coverage problems through evolutionary algorithms. Aerosp. Sci. Technol. 39, 331-337. https://doi.org/ 10.1016/j.ast.2014.09.017.

Casanova, D., Avendano, M.E., Mortari, D., 2014b. Design of flower constellations using necklaces. IEEE Trans. Aerosp. Electron. Syst. 50 (2), 1347-1358. https://doi.org/10.1109/TAES.2014.120269.

Davis, J.J., Avendaño, M.E., Mortari, D., 2013. The 3-d lattice theory of flower constellations. Celestial Mech. Dynam. Astron. 116 (4), 339356. https://doi.org/10.1007/s10569-013-9494-7.

Draim, J.E., 1987. A common-period four-satellite continuous global coverage constellation. J. Guidance, Control, Dynam. 10 (5), 492-499. https://doi.org/10.2514/3.20244.

Lee, S., Avendaño, M., Mortari, D., 2015. Uniform and weighted coverage for large lattice flower constellations. Adv. Astronaut. Sci. $156,3633-3648$.

Lo, M.W., 1999. Satellite-constellation design. Comput. Sci. Eng. 1 (1), 58-67. https://doi.org/10.1109/5992.743623.

Mortari, D., Wilkins, M., Bruccoleri, C., 2004. The Flower Constellations, J. Astronaut. Sci. 52, 107-127.

Mortari, D., Wilkins, M.P., 2008. Flower constellation set theory. part i: Compatibility and phasing. IEEE Trans. Aerosp. Electron. Syst. 44 (3), 953-962. https://doi.org/10.1109/TAES.2008.4655355.

Palmerini, G.B., 1998. Hybrid configurations for satellite constellations. In: Mission Design \& Implementation of Satellite Constellations. Springer, Dordrecht, Netherlands, pp. 81-89. https://doi.org/10.1007/ 978-94-011-5088-0 7 .

Rider, L., 1985. Optimized polar orbit constellations for redundant earth coverage. J. Astronaut. Sci. 33, 147-161.

Speckman, L.E., Lang, T.J., Boyce, W.H., 1990. An analysis of the line of sight vector between two satellites in common altitude circular orbits. In: Astrodynamics Conference, 1990, Portland, OR, paper number AIAA 90-2988-CP. American Institute of Aeronautics and Astronautics Inc, AIAA, Washington DC, USA, pp. 866-874. https://doi.org/ 10.2514/6.1990-2988.

Ulybyshev, Y., 2008. Satellite constellation design for complex coverage. J. Spacecraft Rockets 45 (4), 843-849. https://doi.org/10.2514/1.35369.

Vallado, D.A., 2001. Fundamentals of Astrodynamics and Applications, vol. 12. Microcosm Press and Springer Science \& Business Media, El Segundo, California.

Walker, J., 1984. Satellite Constellations. J. Br. Interplanet. Soc. 37, 559572.

Wilkins, M.P., Mortari, D., 2008. Flower constellation set theory Part ii: secondary paths and equivalency. IEEE Trans. Aerosp. Electron. Syst. 44 (3), 964-976. https://doi.org/10.1109/TAES.2008.4655356. 